\title{
Blunt traumatic carotid artery dissection still a pitfall? The rationale for aggressive screening
}

\author{
K. J. P. van Wessem - J. M. R. Meijer • \\ L. P. H. Leenen - H. B. van der Worp • \\ F. L. Moll • G. J. de Borst
}

Received: 12 February 2010/Accepted: 25 May 2010/Published online: 13 July 2010

(C) The Author(s) 2010. This article is published with open access at Springerlink.com

\begin{abstract}
Introduction The optimal diagnostic strategy for carotid dissection following blunt trauma is yet unclear. The rationale for aggressive screening will be discussed based on a consecutive case series of blunt traumatic carotid artery dissection (CAD).

Materials and methods Five patients admitted to our level I trauma center developed severe complications as a consequence of blunt traumatic CAD. The diagnosis of CAD was delayed in all five patients until serious cerebral ischemia occurred. Despite the current awareness that CAD can result from blunt trauma, this type of injury is often overlooked. Clinical and radiological advances have considerably increased the knowledge of incidence and underlying mechanisms of traumatic CAD. This could have implications for case identification and the evaluation of treatment strategies in clinical trials in the future.

Conclusion Screening may increase the rate of early CAD diagnosis, but it is unclear if screening will also result in early detection of a treatable lesion. Trials have to provide the answer to whether initiating therapy will lead
\end{abstract}

K. J. P. van Wessem · L. P. H. Leenen

Department of Trauma Surgery, University Medical Centre

Utrecht, Utrecht, The Netherlands

J. M. R. Meijer · F. L. Moll · G. J. de Borst $(\bowtie)$

Department of Vascular Surgery,

University Medical Centre Utrecht, G 04.129,

PO Box 85500, 3508 GA Utrecht, The Netherlands

e-mail: G.J.deBorst-2@umcutrecht.nl

H. B. van der Worp

Department of Neurology,

Rudolf Magnus Institute of Neuroscience,

University Medical Centre Utrecht,

Utrecht, The Netherlands to improvements in the outcome in traumatic CAD. We therefore believe that screening is a basic condition for initiation of future clinical trials.

Keywords Blunt · Traumatic - Carotid · Artery · Dissection · Screening

\section{Introduction}

Traumatic carotid artery dissection (CAD) has long been considered a curiosity, but increasingly safe neurovascular imaging techniques of the extra- and intracranial vasculature have indicated that this is a relatively frequent and underestimated cause of stroke. The incidence of CAD among all patients with blunt trauma is estimated at $0.08-1.2 \%[1-8]$, but is considerably higher in patients with certain risk factors. CAD may lead to ischemic stroke and subsequent disability or death. Case fatality has been reported in up to $31 \%$ of the patients with $\mathrm{CAD}$, and severe disability in up to $56 \%$ of the survivors $[9,10]$. CAD not only affects early neurological outcome, but may have severe implications for long-term outcome as well. Unfortunately, most studies that have reported CAD after blunt trauma have had methodological problems, and few of them have presented complete accountability for all patients.

To prevent further complications, early recognition of this injury is important $[4,10,11]$. A critical factor that hampers early diagnosis is the delay between the dissection and the onset of neurological symptoms. Therefore, some authors have proposed early aggressive screening of severely injured patients to detect occult carotid artery dissections [10-13]. Despite several reports on this topic, it is still unclear as to whether aggressive screening is 
beneficial. Screening leads to a major consumption of hospital resources; it is time-consuming, expensive, requires substantial experience from the screener, and probably has a relatively low yield [14]. Furthermore, the natural history of asymptomatic CAD is unknown, and so the most appropriate medical and invasive treatment strategies are still a source of controversy. Modified screening strategies, as advocated by Biffl et al. [13], have been shown to identify only the most likely candidates, thereby increasing the yield from screening and lowering the impact on resources.

We report five patients in whom the diagnosis of CAD was delayed, which may have had a negative effect on functional outcome. The rationale for CAD screening following blunt trauma is discussed.

\section{Patient 1}

A 20-year-old woman presented at our emergency department (ED) following a high-speed car incident against a tree. She was hemodynamically stable, alert, and had no focal neurological deficit. She was diagnosed with a mild bilateral subdural hematoma and fractures of her left zygoma and mandible, right clavicle, and right femur. She was scheduled for operative treatment on her mandibular and femoral fractures for the next day.

During the night she developed respiratory insufficiency due to a lung contusion, after which she was transferred to our intensive care unit (ICU) and intubated. Her Glasgow Coma Scale (GCS) at that time was E3M6V3. A cranial CT was repeated twice that night but did not reveal a plausible explanation for her decreased consciousness. As scheduled, her femur and mandible were operated on the next day. Postoperatively, her consciousness did not improve. At this time, a second evaluation of her previous cranial CT scans and a new CT scan showed a cervical condyle (C0) fracture, and a vertebral artery dissection was then considered. Duplex ultrasound showed no indication of a vertebral artery dissection, but did reveal a hemodynamically significant stenosis in the left internal carotid artery (ICA). Cranial and cervical CT, CT angiography (CTA) and CT perfusion confirmed the dissection of the left ICA (Fig. 1a, b) and demonstrated a severe perfusion deficit of almost the complete territories of the left anterior and middle cerebral arteries and early signs of left middle cerebral artery infarction. CTA showed good collateral circulation via the circle of Willis. Because of the time delay between trauma and the diagnosis of a dissection, the adequate collateral circulation, and probably irreversible cerebral damage, endovascular therapy was not performed, but antiplatelet therapy was started. She was extubated after 11 days, and transferred to the neurological ward with a right hemiparalysis, where she stayed for another six weeks. Ten
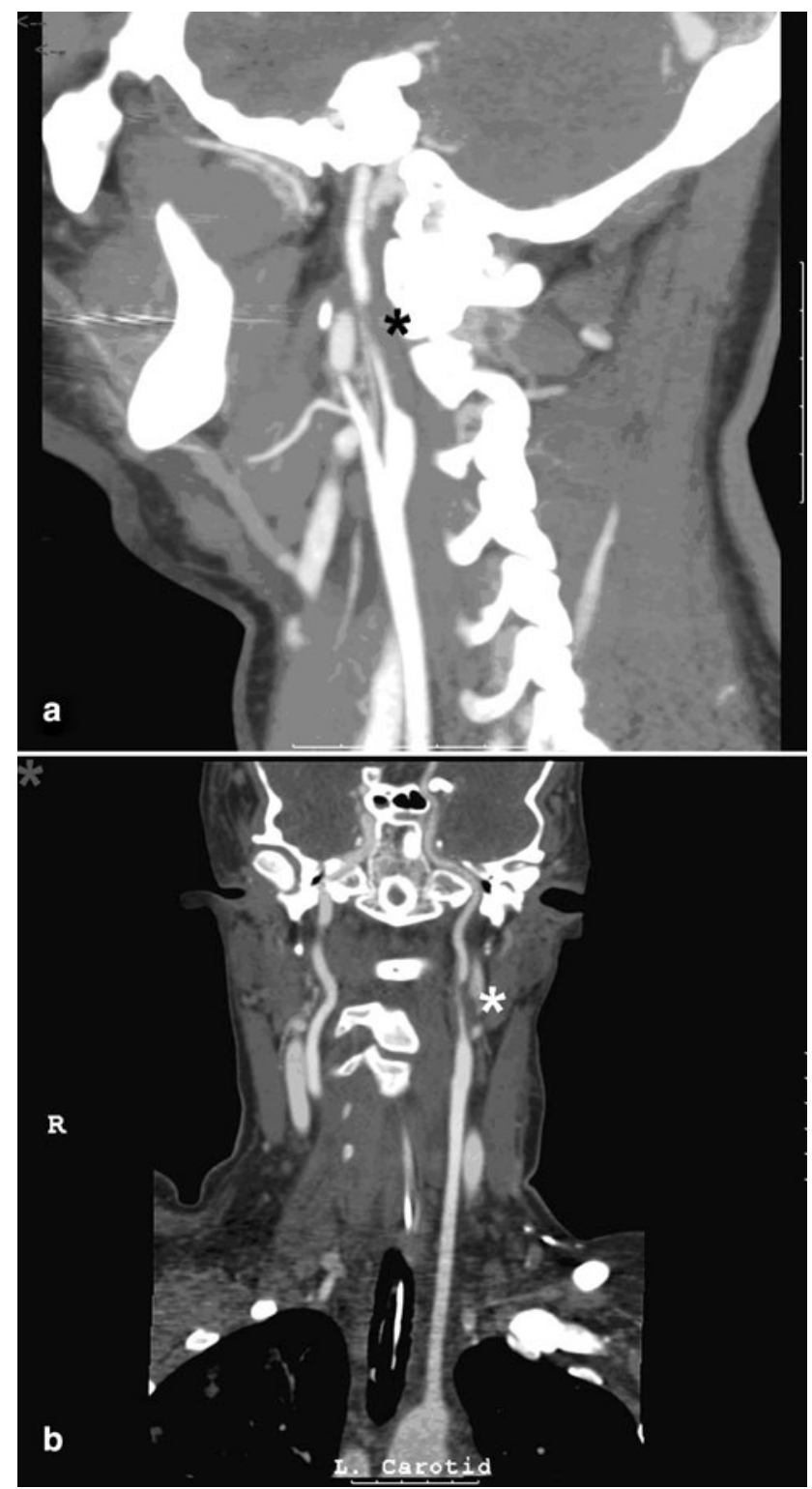

Fig. 1 CTA: images show a subtotal stenosis of the left ICA with a suspected dissection, as indicated by the asterisk

months after the accident she had a mild right-sided hemiparesis and persistent cognitive problems.

\section{Patient 2}

A 49-year-old woman had a high-speed motor cycle accident. On arrival in ED her vital signs were stable and her GCS score was E3M5V4. Screening revealed a sensory loss from her umbilicus downwards and a paralysis of both legs. Other diagnoses were: right temporal lobe contusion with a large hematoma, lung contusion, and multiple rib fractures on the left side for which she received a thoracic drain, a fracture of her left scapula and left humeral head, and multiple fractures of her spine at the cervical and 

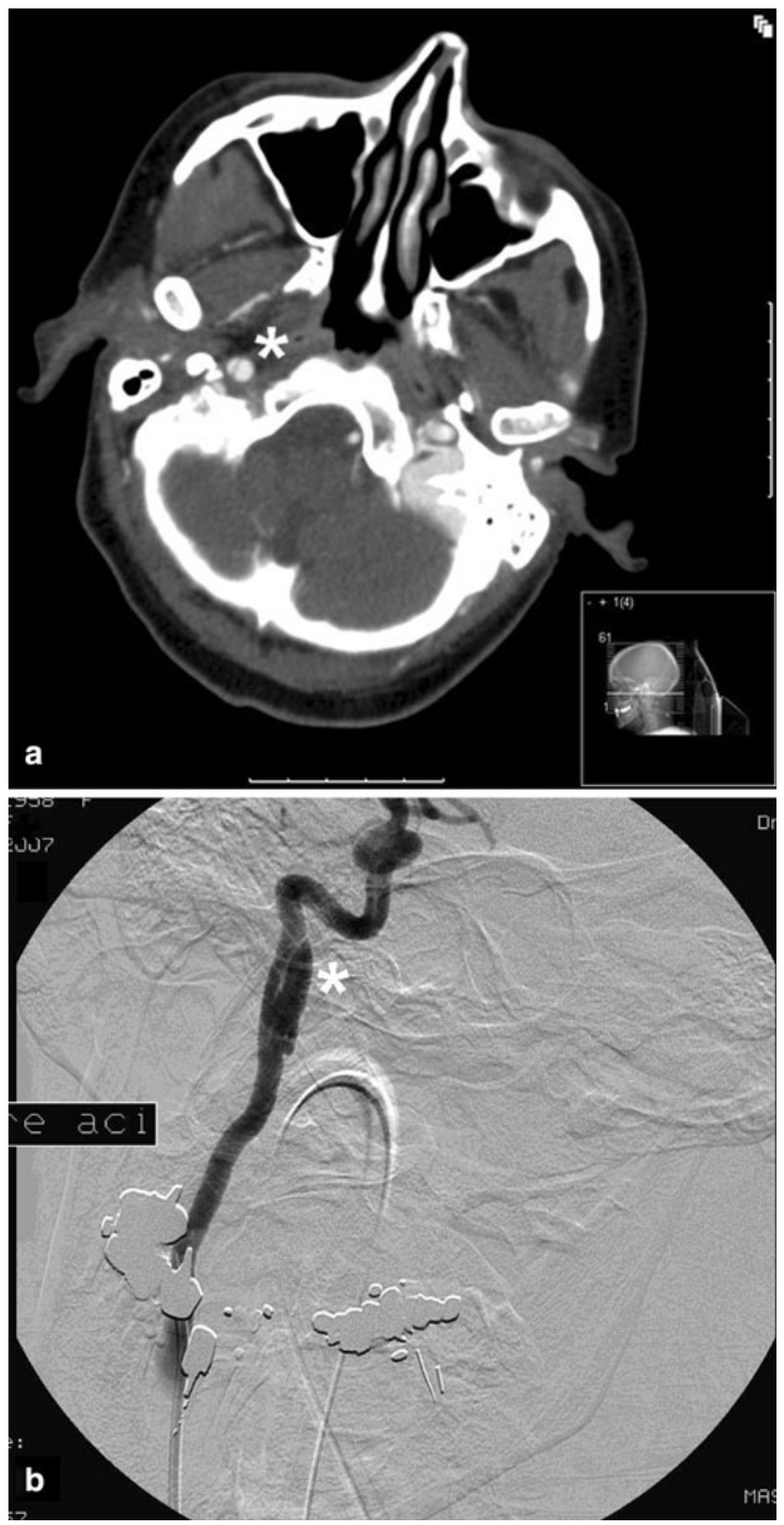

Fig. 2 a CTA: just below the level of the siphon there is a double lumen visible in the right carotid artery with a suspected traumatic dissection of the ICA (asterisk). b Angiography confirms the traumatic ICA dissection (asterisk)

thoracic level. Because of the reduced level of consciousness, another cerebral CT scan was performed, which showed a larger hematoma and increased edema of the right temporal lobe. The hematoma was surgically evacuated and an intracranial pressure (ICP) monitor was introduced. After a relatively stable period of 16 days, spondylodesis of the fourth and fifth thoracic vertebrae was performed, and a tracheotomy after 23 days. She was transferred to the medium-care facility five days after. She made a good recovery and was discharged one month later.
In the outpatient clinic she received a cranial CTA showing a dissection of the right extracranial ICA just below the level of the mastoid (Fig. 2a, b). This diagnosis was confirmed with conventional angiography two months after the accident. She received low-molecular-weight heparin for the prevention of thromboembolic events.

\section{Patient 3}

A 19-year-old man with a history of severe traumatic head and facial injury seven years earlier hit a tree with his motorbike at high speed. At the scene, his vital signs were stable, but he was intubated because of a GCS of E1M4V1. After initial assessment in ED, the diagnoses were: a depressed left temporal fracture, multiple small traumatic intracerebral hematomas, a bilateral mastoid and left sphenoidal, mandibular and clavicular fracture, and a lung contusion. Because of a blood pressure difference between the left and right arm, a CTA of the aorta was performed which showed no rupture or dissection of the thoracic aorta. The patient went to the operating room and an ICP monitor was inserted. Four days later, his mandibular fracture was stabilized. Because of continuous low ICPs, the ICP monitor was removed after six days. However, seven days after the injury the patient developed episodes with bilateral spontaneous stretching of both arms. A cerebral CT scan showed a progression of the left frontal hematoma and an intracerebral hematoma in the left temporal lobe. His pupils became wide and unresponsive to light, with an unchanged GCS score of E1M5V tube and a right hemiparesis. A new $\mathrm{CT}$ scan (eight days after the accident) showed an infarction in the left anterior and middle cerebral arteries. His GCS remained E1M5V tube with a hypertonic hemiplegia on the right side. Treatment focused on maintaining a mean arterial pressure of $>80 \mathrm{~mm} \mathrm{Hg}$. The next day, a CTA showed an ICA dissection from the carotid bifurcation to the siphon.

He was treated with aspirin for six months. Five weeks after the accident the patient was discharged to a nursing home with a GCS of E2M5, dysphasia, and a severe right hemiparesis.

\section{Patient 4}

A 19-year-old man hit a tree with his car at high speed. He was intubated because of a GCS of E1M1V1. He was diagnosed with fractures of the mandible, maxilla, orbita, zygoma, and frontal sinus on the left side, bilateral temporal bone fractures, a cervical condyle ( $\mathrm{C} 0$ ) fracture, and a skull base fracture extending into the carotid siphon on the left side. The patient received a thoracic drain for a right pneumothorax with sternal and bilateral rib fractures. Because of his reduced consciousness and skull fractures, 
cranial and cervical CT and CTA were performed $18 \mathrm{~h}$ post-surgery, which showed a left ICA dissection stretching into the siphon with infarction of the left middle cerebral artery territory. He was treated with aspirin because of the anatomical location of the dissection, and additional injuries precluding anticoagulation therapy.

However, his neurological status deteriorated in the following $48 \mathrm{~h}$. Repeated cranial CT scanning showed the extension of his infarction into the territory of the left anterior cerebral artery with compression of the left ventricle. Further neurological deterioration was observed in the absence of additional treatment options. He was declared clinically dead 14 days after the trauma and accepted for a non-heart-beating donor procedure.

\section{Patient 5}

A 53-year-old male driver was admitted to a level 2 trauma center after a head-on collision with a truck. He was hemodynamically stable with maximal GCS. Other than bilateral $\mathrm{C} 0$ fractures, he had no other injuries. He was transferred to our center for halo frame application almost $18 \mathrm{~h}$ after initial hospital admission. During halo frame-fitting his GCS suddenly decreased to eight. Physical examination showed a right hemiparesis and Babinski reflex on the right side. CT of brain and neck showed a large infarction in the left medial cerebral artery area based on an occlusion of the left ICA based on a dissection of the proximal ICA (Fig. 3a, b). The dissection was inaccessible to surgery due to the extension of the occlusion into the brain (Fig. 3). He was started on antiplatelet therapy. To date, he has a slightly improved right hemiparesis and aphasia.

\section{Discussion}

In our level I university trauma center, approximately 300 multitrauma patients (Injury Severity Score $>15$ ) are treated every year. Urgent screening for CAD in all patients with blunt craniocervical trauma is not performed as yet due to both logistical reasons and an absence of proven effective treatment strategies. The five cases presented here highlight the difficulties involved in diagnosing traumatic CAD without a screening protocol. All of our patients had a CAD-related stroke, and two became symptomatic within $24 \mathrm{~h}$ of their injuries (patient 4 and 5). The remaining three exhibited delayed presentation of symptoms (patients 1,2, and 3 presented, respectively, 24 h, 6 days, and 1 month after injury). Our cases demonstrate that CAD can easily be missed during the initial trauma survey in multiply injured patients, especially in cases of additional traumatic brain injury. Knowledge of the mechanism and risk factors of a traumatic CAD is important because it could have consequences for the treatment of these patients.
Fig. 3 CTA: a narrowing of the proximal left ICA ending in an occlusion (asterisk). This image can be explained by a dissection of the proximal ICA
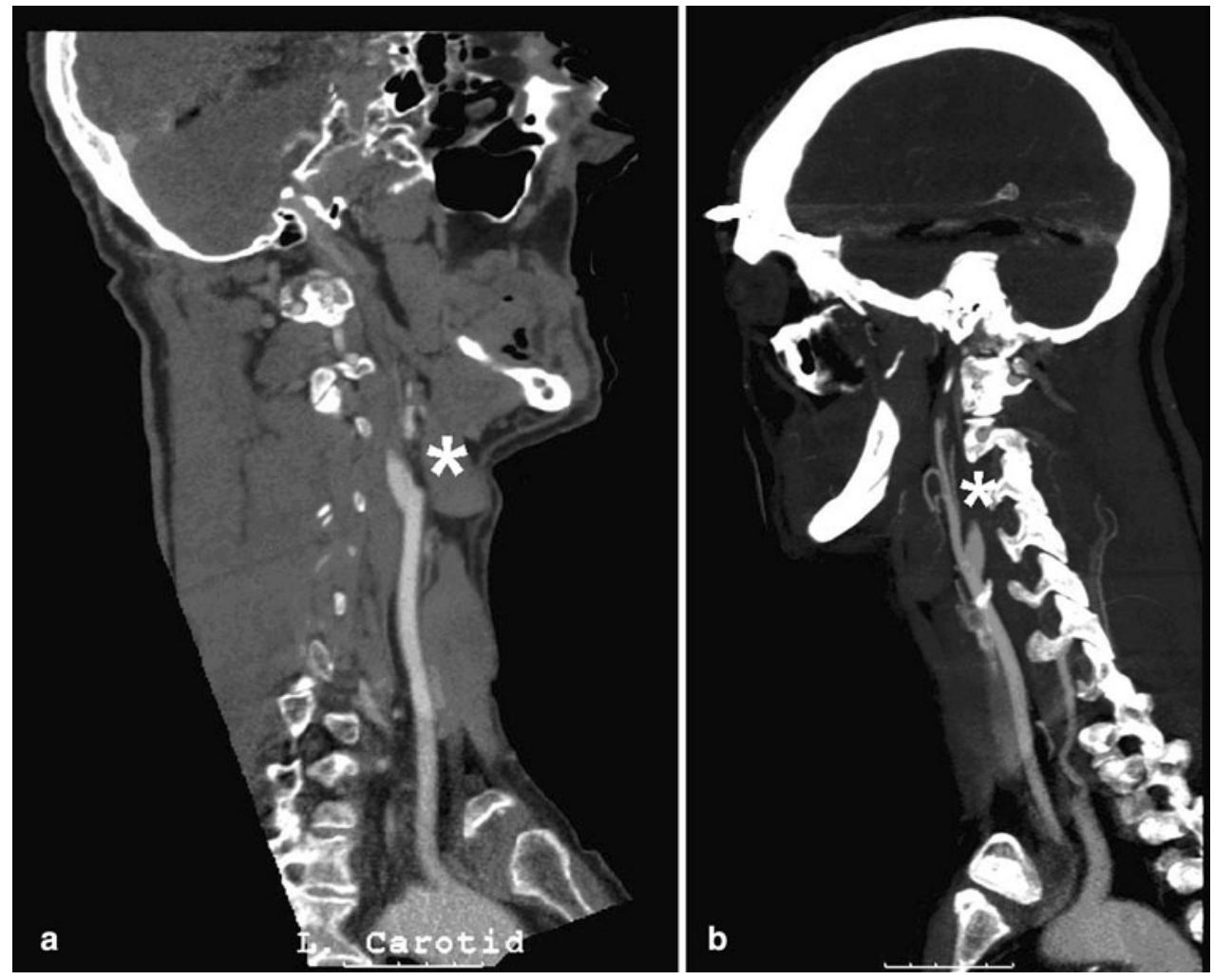


\section{Mechanism}

Dissections start with a tear in the intima/media layer of the vessel wall, after which a hematoma develops. Transient ischemic attacks or ischemic strokes after CAD may occur because of thromboembolism, or, in less frequent cases, artery occlusion due to the hematoma in the vessel wall $[4$, $6,15]$. Dissection can also progress in the subintimal or subadventitial layer, leading to local compression resulting in a loss of function of the adjacent cranial nerves, presenting as Horner syndrome (resulting from periarterial sympathetic plexus disruption), facial nerve palsy, or hypoglossal palsy $[1,14,15]$.

Traumatic causes of a CAD are: (1) direct trauma to the artery, (2) hyperextension-rotation, (3) blunt intraoral trauma, (4) skull base and mandible fractures, and (5) combination of head-thorax trauma with overstretching of the carotid artery $[4,16]$. A specific risk factor for the occurrence of CAD is therefore a trauma in which there is a serious cervical hyperextension, particularly in combination with complex and dislocated midfacial or mandible fractures, a GCS $\leq 6$, closed head injury, skull base fractures involving the carotid canal or fracture of the petrous bone, Le Fort II or III fracture, or a cervical spine fracture (Table 1) $[3-5,9,16,17]$. All of our patients presented with a combination of these CAD risk factors, and all had facial fractures and/or cervical spine fractures.

\section{Clinical symptoms}

Typical symptoms of CAD are pain in the ipsilateral face or neck, followed by contralateral weakness or sensory loss. CAD should also be considered when confronted with a hematoma in the neck or a so-called "seatbelt sign," a cervical bruit in patients younger than 50 years, ptosis and

Table 1 Denver screening criteria for blunt cerebrovascular injury (BCI) [13]

Symptoms and signs
Intraoral arterial hemorrhage
Cervical bruit in patient $<50$ year
Expanding cervical hematoma
Focal neurological deficit
Neurological examinations incongruous with head CT scan findings
Stroke on secondary CT scan
Risk factors
High-energy transfer mechanism with: Le Fort II or III fracture
Cervical spine fracture patterns: subluxation, fractures extending
into the transverse foramen transversarium, fractures of C1-C3
Skull base fracture with carotid canal involvement
Diffuse axonal injury with a Glasgow Coma Scale (GCS) score $<6$
Near-hanging with anoxic brain injury

pupillary asymmetry (ipsilateral Horner's syndrome), a hemorrhage of arterial origin in mouth, nose, or ear, or wounds in the trajectory of the carotid artery $[1,3-5,8,9$, 16]. However, multiply injured patients often cannot express pain due to a lower level of consciousness or a distracting injury.

The interval between blunt cervical injury and (neurological) deficits can be periods of hours, days, or even months, like in our five patients $[6,15,18-20]$. This delay between the moment of dissection and the occurrence of focal neurological symptoms is the critical factor that makes diagnosis a demanding task. Further, it is not at all clear that early detection would have found a treatable lesion after a stroke that is clinically evident hours or days after the injury. Reports of strokes developing after initial negative screening have been made [20]. On the other hand, the time interval between injury and onset of symptoms offers the possibility of screening for CAD and initiating therapy before the neurological symptoms become clear.

\section{Screening}

The challenge is to identify patients with CAD before the onset of neurological complications. Several authors have proposed that liberal screening for blunt carotid and vertebral artery injuries is justified [10, 11, 16, 21], although not all authors were convinced that early detection by screening would also lead to better outcomes [14, 20]. Cothren et al. [11] showed that a screening programme for traumatic CAD was cost-effective since it reduced ischemic strokes and thus costs due to long-term disability. Furthermore, although intracranial dissections are frequently detected and have relatively poor outcomes, extracranial dissections are detected more frequently and stand to benefit from early detection because they are surgically accessible. If early diagnosis of CAD is essential, then who should undergo imaging for screening? It is definitely not advocated to screen for CAD in every trauma patient because of the increased exposure to radiation and its low cost-effectiveness. The yield of screening is probably greater if screening criteria based on a set of risk factors are used (Table 1) [13, 16, 21]. Aggressive screening using these strict criteria identified CAD in $30 \%$ of those screened $[17,26]$. The most efficient type of imaging is yet to be determined [13].

\section{Imaging}

Noninvasive imaging, such as CTA, now allows increasingly rapid and safe evaluation of the presence of CAD, although at the cost of some degree of accuracy [8]. The gold standard for diagnosing CAD is still conventional 
angiography. Besides being expensive and more difficult to obtain, angiography is associated with a complication rate of $1-2 \%$ [4]. In the patients reported here, CTA was used. Trauma patients often already have an indication for CT scanning, and CTA can be a part of this modality. Miller et al. [8] found a sensitivity and specificity of 47 and $99 \%$, respectively, for CTA. As stated above, by using the screening protocol (Table 1) for blunt trauma patients, the diagnostic yield can be enhanced, with sixteen-slice multidetector CTA providing the best results so far $[8,22]$. However, in our department, as in most trauma centers these days, 128-slice CTA is available, and further upgrades to and development of CT technology will probably provide quicker and more accurate diagnosis. Magnetic resonance angiography (MRA) is not available around the clock in all hospitals, and can be difficult in ventilated patients. Duplex ultrasonography (US) can be used as a quick, noninvasive screening tool, and is easy to implement on the ICU. However, duplex US is not available 24/7 in most hospitals, is less reliable in inexperienced hands, is difficult to interpret if there is a hematoma in the neck, and is less reliable for locating dissections near the skull base [4]. When duplex US leads to suspicion of a dissection, additional CTA, MRA, or angiography should be obtained. In our daily practice, CTA has virtually replaced angiography in CAD diagnosis. Only if the diagnosis remains unclear is angiography performed.

Biffl et al. [25] proposed imaging-based criteria to define high-risk arterial injury patterns for stroke and mortality following CAD, using a five-grade classification system (Table 2). Based on angiographic data, the risk of stroke increased from three percent (grade I) to one hundred percent in grade IV, and mortality accordingly increased from eleven to one hundred percent. This classification system potentially provides an excellent way to classify lesion type, bearing in mind that is was designed for conventional angiography.

\section{Management of CAD}

Management of CAD due to blunt trauma remains controversial. Treatment options include observation,

Table 2 Blunt carotid and vertebral arterial injury grade [1]

\begin{tabular}{ll}
\hline Grade I & $\begin{array}{c}\text { Luminal irregularity or dissection } \\
\text { with }<25 \% \text { luminal narrowing } \\
\text { Dissection or intramural hematoma } \\
\text { with } \geq 25 \% \text { luminal narrowing, } \\
\text { an intraluminal thrombus or raised intima flap }\end{array}$ \\
Grade III & Pseudoaneurysm \\
Grade IV & Transection with free extravasation \\
Grade V & Occlusion and transection with extravasation \\
\hline
\end{tabular}

antithrombotic therapy, and endovascular stenting [2, 7, 21, $23,24]$. It has been suggested that with the help of an angiography-based classification system, it may be possible to provide an individualized therapy [7]. However, the use of a classification system has not yet proven to result in a better outcome [9, 14].

It has also been suggested that early recognition of CAD and initiation of treatment with anticoagulant or antiplatelet therapy reduces the number of cerebral ischemic events and improves outcome [7,11], but this also remains to be proven. Furthermore, the type (antiplatelet versus anticoagulant agents) and duration of antithrombotic therapy remain controversial [27]. Arthurs et al. reviewed the literature on treatment outcomes for blunt cerebrovascular injury utilizing heparin, antiplatelet agents, and no antithrombotic therapy. They concluded that treatment reduces the neurological morbidity and mortality associated with BCVI. It remains less clear whether patients should be treated with heparin, aspirin, adenosine diphosphate receptor inhibitors, or a combination therapy.

Unfortunately, patients who sustain blunt carotid injuries typically have associated closed head injuries, solid organ injuries, or pelvic fractures that prevent the use of early anticoagulation [27].

Literature on the stenting of traumatic CAD is still limited to small series with relatively short follow-up [7, $23,24]$. A proportion of the dissections occur at the skull base or intracranially, and are therefore inaccessible to surgical or endovascular therapy. However, recent studies of stenting for CAD show excellent early and one-year patency rates and a low major adverse event rate [24]. Further studies are warranted to define the role of this treatment modality in the setting of treatment of blunt carotid injury.

Some authors have stated that screening is not useful because it will not change therapeutical options, leaving antiplatelet therapy as the only therapeutical option [20, 21]. Clearly, if all trauma patients were to receive aspirin, many would be overtreated and incur an increased risk of bleeding. The usefulness of liberal screening could also be questioned based on a recent report demonstrating that nearly one-third of patients with cerebrovascular injuries are probably not candidates for endovascular or antithrombotic therapy due to intracranial lesions or concomitant injuries, as in the third and fourth cases we presented here [20]. However, easily accessible extracranial lesions occur more frequently than intracranial lesions and may benefit from early detection $[11,23]$.

A limitation of the present report is that we were not informed about the total number of patients in whom a dissection was missed due to a lack of routine CTA screening in our hospital. In the literature, an incidence of $\mathrm{CAD}$ of $1 \%$ in blunt trauma admissions was suggested. On 
the other hand, all five patients described above met the criteria for CAD screening mentioned in Table 1 and could possibly have been offered earlier treatment, but it remains uncertain as to whether their outcomes would have been better had a more aggressive screening and treatment policy been adopted. The patients received only medical treatment because of surgically or endovascularly inaccessible lesions, and especially because of delays between trauma and diagnosis. Also, because the cases presented here are major trauma cases, the role that other neurological injuries may have played in these patients' adverse outcomes is unclear.

\section{Conclusion}

Traumatic CAD is probably still underdiagnosed. Knowledge of whether to screen for CAD or not is still evolving. The use of a widely applied screening policy and a uniform classification system may increase the rate of early CAD diagnosis, facilitate improvements in imaging modalities, and increase tailormade therapies. However, the benefit this policy may have remains unclear, since diagnosing CAD in some patients would probably not have altered outcomes. Further, it is still not clear if screening also results in early detection of a treatable lesion.

Prospective randomized clinical trials need to be designed to answer the question of whether initiating therapy early will lead to improvements in outcome in traumatic $\mathrm{CAD}$. We therefore believe that screening is a basic condition for initiating future clinical trials.

\section{Conflict of interest statement None.}

Open Access This article is distributed under the terms of the Creative Commons Attribution Noncommercial License which permits any noncommercial use, distribution, and reproduction in any medium, provided the original author(s) and source are credited.

\section{References}

1. Biffl WL, Moore EE, Offner PJ, Burch JM. Blunt carotid and vertebral arterial injuries. World J Surg. 2001;25:1036-43.

2. Cothren CC, Moore EE, Biffl WL, Ciesla DJ, Ray CE Jr, Johnson JL, Moore JB, Burch JM. Anticoagulation is the golden standard therapy for blunt carotid injuries to reduce stroke rate. Arch Surgery. 2004;139:540-6.

3. Biffl WL. Diagnosis of blunt cerebrovascular injuries. Curr Opin Crit Care. 2003;9:530-4.

4. Miller PR, Fabian TC, Croce MA, Cagiannos CC, Williams JS, Vang M, Qaisi WG, Felker RE, Timmons SD. Prospective screening for blunt cerebrovascular injuries: analysis of diagnostic modalities and outcomes. Ann Surg. 2002;236:386-95.

5. De Borst GJ, Slieker MG, Monteiro LM, Moll FL, Braun KPJ. Bilateral traumatic carotid artery dissection in a child. Pediatr Neurol. 2006;34:408-11.
6. Kraus RR, Bergstein JM, DeBord JR. Diagnosis, treatment and outcome of blunt carotid arterial injuries. Am J Surg. 1999;178:190-3.

7. Edwards NM, Fabian TC, Claridge JA, Timmons SD, Fischer PE, Croce MA. Antithrombotic therapy and endovascular stents are effective treatment for blunt carotid injuries: results from longterm followup. J Am Coll Surg. 2007;204:1007-13.

8. Berne JD, Keuland KS, Villarreal DH, McGovern TM, Rowe SA, Norwood SH. Sixteen-slice multi-detector computed tomographic angiography improves the accuracy of screening for blunt cerebrovascular injury. J Trauma. 2006;60:1204-9. (discussion 1209-1210).

9. Hughes KM, Collier B, Greene KA, Kurek S. Traumatic carotid artery dissection: a significant incidental finding. Am Surg. 2000;66:1023-7.

10. Kerwin AJ, Bynoe RP, Murray J, Hudson ER, Close TP, Gifford RR, Carson KW, Smith LP, Bell RM. Liberalized screening for blunt carotid and vertebral artery injuries is justified. J Trauma. 2001;51:308-14.

11. Cothren CC, Moore EE, Ray CE, Ciesla DJ, Burch JM. Screening for blunt cerebrovascular injuries is cost-effective. Am J Surg. 2005;190:849-54.

12. Biffl WL, Moore EE, Ryu RK, Offner PJ, Novak Z, Coldwell DM, Franciose RJ, Burch JM. The unrecognized epidemic of blunt carotid internal arterial injuries. Ann Surg. 1998;228:462-70.

13. Biffl WL, Moore EE, Offner PJ, Brega KE, Franciose RJ, Elliott JP, Burch JM. Optimizing screening for blunt cerebrovascular injuries. Am J Surg. 1999;78:517-22.

14. Mayberry JC, Brown CV, Mullins RJ, Velmahos GC. Blunt carotid artery injury: the futility of aggressive screening and diagnosis. Arch Surg. 2004;139:609-12. (discussion 612-613).

15. Crissey MM, Bernstein EF. Delayed presentation of carotid intimal tear following blunt craniocervical trauma. Surgery. 1974;75:543-9.

16. McKevitt EC, Kirkpatrick AW, Vertesi L, Granger R, Simons $\mathrm{RK}$, Chir B. Identifying patients at risk for intracranial and extracranial blunt carotid injuries. Am J Surg. 2002;183:566-70.

17. Cothren CC, Moore EE, Ray CE Jr, Johnson JL, Moore JB, Burch JM. Cervical spine fracture patterns mandating screening to rule out blunt cerebrovascular injury. Surgery. 2007;141:76-82.

18. Pozzati E, Giuliani G, Poppi M, Faenza A. Blunt traumatic carotid dissection with delayed symptoms. Stroke. 1989;20:412-6.

19. Krajewski LP, Hertzer NR. Blunt carotid artery trauma. Ann Surg. 1980;91:341-6.

20. Stein DM, Boswell S, Sliker CW, Lui FY, Scalea TM. Blunt cerebrovascular injuries: does treatment always matter? J Trauma. 2009;66:132-43. (discussion 143-144).

21. Fabian TC, Patton JH Jr, Croce MA, Minard G, Kudsk KA, Pritchard FE. Blunt carotid injury: importance of early diagnosis and anticoagulant therapy. Ann Surg. 1996;223:513-25.

22. Biffl WL, Egglin T, Benedetto B, Gibbs F, Cioffi WG. Sixteenslice computed tomographic angiography is a reliable non-invasive screening test for clinically significant blunt cerebrovascular injuries. J Trauma. 2006;60:745-51. (discussion 751-752).

23. Donas KP, Mayer D, Guber I, Baumgartner R, Genoni M, Lachat M. Endovascular repair of extracranial carotid artery dissection: current status and level of evidence. J Vasc Surg. 2008; $19: 1693-8$

24. DuBose J, Recinos G, Teixeira PG, Inaba K, Demetriades D. Endovascular stenting for the treatment of traumatic internal carotid injuries: expanding experience. J Trauma. 2008;65:1561-6.

25. Biffl WL, Moore EE, Offner PJ, Brega KE, Franciose RJ, Burch JM. Blunt carotid arterial injuries: implications of a new grading scale. J Trauma. 1999;47:845-53. 
26. Kortbeek JB, Al Turki SA, Ali J, Antoine JA, Bouillon B, Brasel $\mathrm{K}$, Brenneman F, Brink PR, Brohi K, Burris D, Burton RA, Chapleau W, Cioffi W, Collet e Silva Fde S, Cooper A, Cortes JA, Eskesen V, Fildes J, Gautam S, Gruen RL, Gross R, Hansen KS, Henny W, Hollands MJ, Hunt RC, Jover Navalon JM, Kaufmann CR, Knudson P, Koestner A, Kosir R, Larsen CF, Livaudais $\mathrm{W}$, Luchette F, Mao P, McVicker JH, Meredith JW, Mock C, Mori ND, Morrow C, Parks SN, Pereira PM, Pogetti RS,
Ravn J, Rhee P, Salomone JP, Schipper IB, Schoettker P, Schreiber MA, Smith RS, Svendsen LB, Taha W, van Wijngaarden-Stephens M, Varga E, Voiglio EJ, Williams D, Winchell RJ, Winter R. Advanced trauma life support, 8th edition, the evidence for change. J Trauma. 2008;64:1638-50.

27. Arthurs ZM, Starnes BW. Blunt carotid and vertebral artery injuries. Injury. 2008;39:1232-41. 